L'HOMME L'Homme

171-172 | 2004

Musique et anthropologie

\title{
Inherent patterns
}

Musiques de l'ancien royaume de Buganda: étude de psychologie cognitive

Gerhard Kubik

\section{OpenEdition}

Journals

Édition électronique

URL : http://journals.openedition.org/lhomme/24906

DOI : 10.4000/lhomme.24906

ISSN : 1953-8103

Éditeur

Éditions de l'EHESS

Édition imprimée

Date de publication : 1 décembre 2004

Pagination : 249-264

ISSN : 0439-4216

Référence électronique

Gerhard Kubik, «Inherent patterns », L'Homme [En ligne], 171-172 | 2004, mis en ligne le 01 janvier

2006, consulté le 19 avril 2019. URL : http://journals.openedition.org/lhomme/24906 ; DOI : 10.4000/ Ihomme.24906

(c) École des hautes études en sciences sociales 


\title{
Inherent patterns \\ Musiques de l'ancien royaume de Buganda: étude de psychologie cognitive
}

\author{
Gerhard Kubik
}

LES FAITS de psychologie de l'audition que j'ai pu étudier en Afrique subsaharienne pendant ces quatre dernières décennies ne permettent pas d'affirmer qu'il existe une "psychologie africaine » spécifique. Ils soulignent au contraire l'unité psychologique de l'humanité. Ils soulignent aussi l'existence de comportements (associés à des phénomènes universels) qui ne sont pas innés, mais acquis. Les réactions humaines sont conditionnées et les concepts qui s'y rattachent sont appris et transmis. C'est pour cela qu'ils ne sont pas attestés partout de la même façon et avec la même intensité. D’un endroit à l'autre, l'apprentissage est variable, et développé diversement, comme sont variables les connaissances qui lui sont liées. Dans certaines cultures musicales, ces connaissances sont même inconnues.

De longues périodes d'isolement géographique ont conduit l'homme à se spécialiser, à poursuivre des orientations particulières pour ce qui est de la technologie, de la régulation sociale, de la création d'institutions et des expressions qui s'y rattachent. C'est le cas, par exemple, dans la musique de cour de l'ancien royaume de Buganda, dans la région des lacs en Afrique orientale - un royaume existant depuis le XIV siècle, d'après la généalogie dynastique et les dates tirées des fouilles archéologiques. Ici, quelques découvertes touchant à la psychologie de l'audition musicale faites par de grands experts du pays nous ont été transmises par le biais de compositions dont la plupart datent de la fin du XVIII et de la première moitié du XIX ${ }^{\mathrm{e}}$ siècle.

Les études récentes au royaume de Buganda ont été réalisées avec l'accord du roi de Buganda, Ssabasajja Ronald Mutebi II, et avec le concours du Fonds zur Förderung der Wissenschaftlichen Forschung, Vienne, dans le contexte de notre projet courant P 15007 sur les anciens royaumes de la région des lacs. Je voudrais remercier en particulier mon collègue Charles Sekintu, historien du Buganda, les musiciens de cour, Albert Ssempeke et son fils, Albert Bisaso, et Moya Aliya Malamusi, anthropologue qui a toujours partagé avec moi les vicissitudes de la recherche sur le terrain. Enfin, je remercie mes collègues français Bernard Lortat-Jacob et Miriam Rovsing Olsen de m'avoir invité à écrire cette contribution et pour la correction de quelques insuffisances inévitables dans ce texte que j’ai rédigé moi-même dans la langue de René Descartes. 
Beaucoup de découvertes scientifiques faites dans l'Afrique précoloniale sont peu connues, par exemple dans le domaine de la métallurgie, de la technologie et de l'acoustique des lamellophones, ou encore de l'écriture idéographique autrefois pratiquée dans l'Est de l'Angola. Transmises localement, ces découvertes scientifiques étaient souvent limitées au cercle des spécialistes, comme en Occident où la physique ou les mathématiques ne sont pas des patrimoines communs, même si, en principe, elles sont accessibles à tous. Pourtant, ces connaissances sont devenues ésotériques parce que l'homme, bousculé par les tempêtes de sa courte vie, ne trouve ni l'intérêt, ni le temps, ni la persévérance de s'en préoccuper.

En un sens, la production de la musique de cour au Buganda était ésotérique, même si cette musique n'était pas exclusive. Les découvertes relatives à sa conception et à sa perception relevaient du domaine des musiciens spécialistes mis à disposition du roi par les clans totémiques du pays, au nombre de cinquante-deux. Mais les manifestations publiques étaient toujours accessibles à tout le monde. Les gens dansaient, chantaient, frappaient dans leurs mains et s'amusaient.

Le facteur culturel consiste en l'accumulation des connaissances et en leur transmission. Certains modes de comportement, acquis et appris, sont associés ensuite à des expressions verbales; celles-ci se servent de la langue locale et entrent dans une terminologie de spécialistes avant d'être transmises de génération en génération. Toutes les cultures du monde ont des configurations spécifiques et représentent toujours une solution temporaire aux tentatives permanentes de réaliser et maintenir un équilibre socio-psychologique.

À partir de cas concrets dans la musique de cour au Buganda, j'ai étudié surtout la fission auditive conduisant à la perception de ce que j’ai appelé des inherent patterns. Ceux-ci relèvent d'une stratégie artistique extrêmement développée.

Ces phénomènes de perception auditive sont universels et absolument indépendants de la "culture» et de la langue maternelle. C'est leur usage dans le domaine de la composition musicale qui nous intéresse ici parce qu'il s'est énormément développé dans différentes formes de musique africaine. Dans certaines régions de l'Afrique, dans des contextes institutionnels particuliers, de grands savants africains du passé ont découvert, examiné, puis appliqué leurs connaissances des réactions caractéristiques de nos appareils perceptifs auditif et visuel. Ces réalisations sont uniques et ne sont pas attestées avec le même raffinement dans d'autres cultures musicales du monde.

\section{Battement encrené ${ }^{1}$}

La musique au Buganda, jouée à la harpe (cf. CD \# 27) ou au xylophone, est très rapide. La pulsation élémentaire renvoyant aux plus petites unités référentielles de temps conceptualisées par les musiciens et les danseurs s'élève à 600 pulsations à la minute [600 bpm]. Cette régulation est toujours présente au Buganda. On peut observer que la vitesse des mouvements pelviens des femmes

1. "Encrené" est un vieux mot de la langue française, selon le Robert, il signifie "faire entrer les dents d'une roue dans les espaces séparant les dents d'une autre roue, à la manière d'un engrenage ". 
et des jeunes filles, le buste restant presque statique, correspond toujours à cette pulsation élémentaire. Des enfants qui sont exposés quotidiennement à une telle culture musicale sont sous l'emprise de ce stimulus. Dans le processus d'endoculturation individuelle, ce comportement perceptif est graduellement intériorisé pour être ensuite projeté sur n’importe quel événement sonore.

Dans une musique fondée sur un tel schéma de référence, il est possible de développer des chaînes d'accents irréguliers, sans que les auditeurs se "perdent ». Cette pulsation élémentaire, qui peut être silencieuse et plus ou moins consciente, n’a pas de structure interne: ni commencement, ni fin, ni accentuation. De ce fait, elle peut connaitre diverses accentuations, mais ces dernières doivent toujours coïncider avec les unités de pulsation élémentaire constitutives de ce réseau interne.

Jouer une telle musique semble exiger une organisation spécifique, une division du travail. En fait, dans la musique de cour au Buganda, on a développé probablement depuis des siècles - un système d'exécution musicale dans lequel la structure compositionnelle est subdivisée en deux moitiés, jouées chacune à demi-vitesse, de sorte que chaque musicien a pour base métronomique une pulsation à 300. C'est comme si l'on était en présence d'une série de chiffres : 1, 2, $3,4,5$ où, en alternance serrée, $1,3,5$ seraient prononcés par un protagoniste, et 2,4 par un autre.

C'est le principe du jeu de xylophone amadinda au Buganda : on joue des séries mélodiques qui se combinent en s'encrenant comme des roues dentées.

Figure I. Amadinda

(nom en langue luganda d'un xylophone sur bananiers comprenant douze lames).

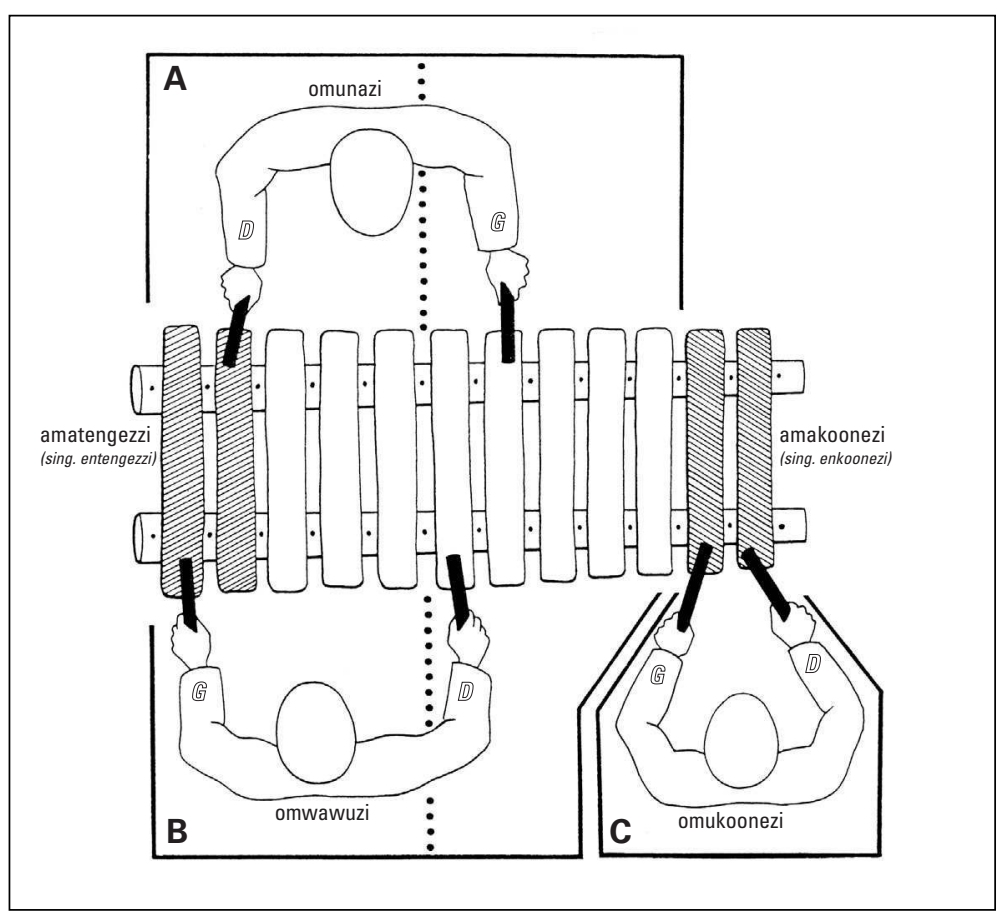


Trois musiciens jouent ensemble. Deux d'entre eux - ceux qui nous intéressent ici - jouent les séries mélodiques de base à encrener; on les appelle en luganda omunazi (celui qui commence à jouer) et omwawuzi (celui qui "divise " la série mélodique du premier, en encrenant après quelques secondes sa propre série).

La musique est ancienne et on dit que la plupart des compositions historiques étaient originalement conçues pour la harpe ennanga (CD \# 27). Ensuite, les séries de sons sur la harpe ont été distribuées entre deux musiciens jouant du xylophone. En effet, le musicien 1 (omunazi) joue la partie de main droite du harpiste cependant que le musicien 2 (omwawuzi) joue la partie de main gauche.

Sur le xylophone, les séries de base sont toujours exécutées en octaves parallèles. Les musiciens se placent en face l'un de l'autre, chacun possédant deux baguettes et jouant ses propres lames en octaves : c'est la première règle d'exécution. La deuxième est l'absence d'improvisation ; ce sont des compositions fixes. On peut, bien sûr, changer l'accentuation et ainsi créer l'apparence d'une variation, mais il est très rare qu'on change quelques notes. L'accord des xylophones dans le Sud de l'Ouganda, de l'amadinda comme du xylophone très ressemblant qu'on joue au Busoga, petit État voisin de l'ancien royaume de Buganda, est toujours équipentatonique. C'est-à-dire que l'octave est divisée en cinq degrés distants d'environ 240 cents, représentés dans la notation de bas en haut (du grave vers l'aigu) avec les chiffres $1,2,3,4,5$. Deux cent quarante cents est l'intervalle standard, mais il y a une marge de tolérance de plus ou moins 10-20 cents. Au Buganda, on aime ce que j'ai appelé des octaves en friction, cela veut dire qu'intentionnellement, on n'accorde jamais en octaves réelles, à 1200 cents exactement. Cela souligne la singularité mélodique des phrases et diminue les effets de fusion acoustique.

Le musicien qui est en charge d'exécuter la série initiale, une tâche qu'on appelle okunaga en luganda (" commencer à frapper, entonner»), joue sa partie en octaves parallèles. À un certain moment, mémorisé à l'avance (nous le marquons avec une flèche dans la transcription qui suit, en Fig. 2), le deuxième musicien doit entrer, encrenant sa propre série, une tâche appelée okwawula, ce qui veut dire: séparer, mettre à part, diviser (également en octaves parallèles). Le terme décrit bien de quoi il s'agit. Cette série à encrener est analogue à la série de base, bien qu'elle soit mélodiquement différente.

Les pièces d'amadinda sont cycliques. L'ensemble répété fonctionne sur la base de 36 unités de pulsation (voir Fig. 2). Donc, le premier musicien commence (en octaves parallèles) avec sa série: 4.5.2.3.3.5. etc. et au moment prévu, le deuxième s'insère dans les frappes du premier, avec 5.4.3.2.4. etc. Les frappes des deux ne coïncident jamais. Dans la notation, le point signifie qu'on ne frappe pas; il équivaut aux silences de la notation occidentale. 
Okunaga

Okwawula

Figure 2. Les deux parties encrenées de Ssematimba ne Kikwabanga (Ouganda, décembre 1959).

Chaque série est redoublée à l'octave et chaque chiffre ou point correspond à une unité de pulsation (CD \# 28 et \# 29).

Il faut savoir que jouer ce morceau à la vitesse individuelle de $300 \mathrm{bpm}$ et bien exécuter en octaves parallèles les deux séries encrenées comme elles sont transcrites exige un apprentissage et beaucoup de pratique. En décembre 1959, à Kampala, j'avais commencé à apprendre à jouer de l'amadinda ${ }^{2}$. Mon professeur était Evaristo Muyinda, musicien de la cour. Les problèmes à résoudre étaient énormes, surtout parce que j'avais supposé, à tort, qu'il m’apprendrait des «formules" rythmiques et leurs combinaisons. Mais mon professeur insistait pour que j'apprenne des séries! Toutefois, le plus grand problème était la compréhension de l'encrenage: comment le conceptualiser? De décembre 1959 à avril 1960, durant la première phase de mon apprentissage, je n'étais pas le seul étudiant d'Evaristo Muyinda. Il enseignait aussi à des garçons, qui étaient tous d'ethnies baganda ou basoga et qui avaient l'avantage de bien parler la langue. Ils avaient également des difficultés, surtout pour mémoriser les séries longues. Mon problème à moi était d'une autre nature. En jouant l'okwawula, par exemple dans la composition Ssematimba ne Kikwabanga (Fig. 2), il me fallait insérer la série 5.4.3.2. etc. au point prévu entre les notes jouées par mon professeur, et garder le tempo. Régulièrement je me trouvais déconnecté au bout de dix secondes. Pour le musicien de jazz que j'étais, c'était humiliant. En outre, je n'arrivais pas à déterminer les raisons de ma difficulté jusqu'au moment où je compris que je cherchais la syncope avec mon partenaire, imaginant une mesure commune.

Mon professeur était aussi désespéré que moi, mais il croyait que mon problème provenait d'un déficit de concentration, ou d'une incapacité à tenir le temps. Enfin, il trouva un remède. Comme il avait constaté que, laissé seul, je jouais ma partie comme il le fallait, il me proposa tout simplement de commencer avec l'okwawula, comme si c'était la première partie, et il faisait entrer l'okunaga au moment approprié.

Ainsi, il n'était plus possible pour moi de faire syncoper ma partie avec la sienne! Prenant mes propres frappes comme ligne référentielle, la combinaison se formait instantanément comme prévu, et je soutenais ma partie de façon détendue. Par un simple artifice didactique, non verbal, Evaristo Muyinda rectifiait la fausse base de mon jeu. Forcé de commencer avec ma série dite encre-

2. De cette époque, j'ai pu noter et conserver en notation chiffrée une centaine de pièces jouées sur deux types de xylophones, des amadinda ou des akadinda. 
nante, sans base à laquelle l'encrener, je la jouais comme je l'avais apprise sans aucune référence extérieure.

Peu à peu je compris que les deux séries à combiner étaient à concevoir sans référence à un beat commun, ni à un jeu de syncope ou de beat loff beat. Chaque série avait une fonction relative à l'autre. Bientôt, je pus constater que ce système cognitif s'appliquait aussi à l'akadinda à 22 lames, le deuxième type de xylophone utilisé dans la musique de cour de Buganda. Ainsi, j’ai pu redécouvrir les observations de Jones (1934) relatives au jeu de tambours ngwayi en Zambie: pour l'akadinda, comme pour le ngwayi, le mode de combinaison est celui d'un triple encrenage.

\section{Inherent patterns}

Une fois compris comment conceptualiser cet encrenage des séries en jouant de l'amadinda, les surprises n'étaient pas terminées. En 1959, lors de mes premières rencontres avec des musiciens de cour de Buganda, je me suis trouvé plusieurs fois exposé à un choc culturel. Je compris qu'Evaristo Muyinda ne m'enseignerait jamais de "rythmes». Il m’apprenait exclusivement à frapper régulièrement des séries de notes sur les lames du xylophone, en octaves parallèles, et ainsi j'appris en quelques semaines les séries okwawula et okunaga d'une douzaine de compositions anciennes.

Heureusement, je n'étais ni rebelle ni prétentieux et ne laissai pas croire que je connaissais tout. J'acceptai l'autorité de mon tuteur, qui dut satisfaire ma curiosité d'une autre façon. Chaque chose qu'il m'enseignait sur le xylophone était identifiée par une terminologie élaborée en luganda, utilisée par les musiciens de la cour. Nous commencions à communiquer avec des notions telles que okunaga, okwawula, okukoonera, omunazi, omwawuzi, amatengezzi, amakoonezi, miyanjo, emiko, okunsengejja, okudaliza, etc. (voir Kubik 1994 : 53-58, pour une introduction). Ainsi ai-je été conduit, dès le début de mes recherches, à pratiquer une approche "émique" m'invitant à comprendre cette musique d'un point de vue interne.

Dès que j'eus mémorisé quelques-unes de ces séries, je constatai en jouant que, du moment que la combinaison de nos deux parties était correcte, la mienne avait tendance à disparaître. Bien sûr, elle était physiquement présente, mais la mélodie de ma série était submergée par une réalité d'une immense complexité qui avait tendance à osciller à la façon d'une illusion d'optique. J'observai que plusieurs lignes mélodico-rythmiques surgissaient de notre combinaison et j'étais sûr que ni mon partenaire ni moi-même ne les avions jouées. D'abord je pensai que c'était un effet provoqué par nos accentuations, mais assez vite, je dus reconnaître que c'était plus que cela. Je mis ce phénomène en rapport avec les illusions d'optique mises en évidence par la Gestalt Psychology (Christian von Ehrenfels 1890, Wolfgang Köhler 1929, et Kurt Koffka 1935).

Je commençai à donner un nom à ces phrases qui surgissaient de notre jeu: c'étaient des « rythmes subjectifs ou inhérents " (Kubik 1960 : 12), et je caractérisai 
ma découverte comme un phénomène de pure gestalt. Plus tard, je simplifiai la terminologie, parlant de inherent patterns ou simplement "d'effet I. P. » parce qu'il ne s'agissait pas seulement de "rythmes », mais de résultantes mélodico-rythmiques. Il y avait là un aspect objectif et un aspect subjectif. L'aspect objectif était que ces inherent patterns étaient indispensables à la structure de la composition et probablement intentionnels; il ne s'agissait pas d'hallucinations auditives. L'aspect subjectif tenait au fait que personne ne les avait joués directement et qu'ils existaient seulement dans la perception humaine sous forme de gestalt (Kubik 1960 : 13).

Mais il fallait en faire la preuve. Il fallait d'abord réaliser un enregistrement pour être absolument sûr de ce que notre perception auditive créait ces patterns à partir de la combinaison de okunaga et okwawula. Et il fallait que je joue moimême une partie de la composition pour être assuré qu'elle avait vraiment disparu et que ce que nous avions effectivement joué ne correspondait pas à ce que livrait l'enregistrement. Cette occasion ne se produisit qu'en février 1960, à l'Uganda Museum, où Evaristo Muyinda enseignait, le jour où l'on installa la jolie amadinda dans le jardin pour éviter l'acoustique des salles du musée. Je frappai la série okunaga, Evaristo Muyinda, l'okwawula, et un collègue, Ismail Obaloker, l'okukoonera (CD \# 30). Contrairement à ses deux autres partenaires, le musicien 3, appelé omukoonezi du fait de sa fonction dans le groupe d'exécutants, ne frappe que sur les deux lames les plus aiguës du xylophone, et il joue une phrase rythmiquement très complexe.

Mon professeur me donna l'okunaga pour l'enregistrement et, comme d'habitude, chacun s'occupa principalement de son propre rôle dans l'ensemble, sans porter beaucoup d'attention aux autres (cela vaut surtout pour les débutants). Mais il y eut une petite crise d'une autre nature : le directeur du musée découvrit que nous avions mis le xylophone dans le jardin et il était résolument contre une telle entreprise. Nous ne pûmes donc enregistrer qu'un seul morceau, Ssematimba ne Kikwabanga. Cependant, ce court enregistrement a permis de répondre à mes questions les plus urgentes.

Dans notre combinaison surgirent des phrases mélodico-rythmiques que personne d'entre nous n'avait jouées. Je dus poursuivre mes investigations sur le papier! Je réalisai des transcriptions, et ces phrases étaient manifestes, alors même que personne ne les avait jouées. Elles étaient cachées dans la composition, mais comme une image auditive et non comme une véritable réalisation.

La transcription mise en annexe de cet article (Fig. 3), montre l'ensemble de l'organisation de la composition Ssematimba ne Kikwabanga. Il s'agit d'un enregistrement réalisé quelques années plus tard, en 1967, à Kampala, avec un autre musicien de cour, Albert Ssempeke, qui jouait alors avec son frère et son fils. Depuis la mort de mon professeur Evaristo Muyinda, survenue en 1997, c'est lui qui représente aujourd'hui la tradition. Dans l'enregistrement (CD \# 31), transcrit ici, il explique également les différentes parties de cette composition ${ }^{3}$.

3. Cet enregistrement date de novembre 1967, alors que les photos sont de février 2000, moment où je pus reprendre mes recherches dans le royaume de Buganda. Il s'agit d'un petit .../... 
Ssematimba ne Kikwabanga est une composition très célèbre. On ne chante pas en jouant de l'amadinda, mais j'ai quand même transcrit la partie chantée dans la figure 3. Le thème chanté, lorsque la composition est jouée par exemple à la harpe, se réfere à deux princes nommés Ssematimba et Kikwabanga, morts dans une bataille à la fin du XVIII ${ }^{\mathrm{e}}$ ou au début du XIXe siècle. Ils étaient très confiants dans leur victoire et pensaient revenir célèbres au village. Avant leur départ, ils enjoignirent leurs compatriotes de sacrifier et de préparer des chèvres pour leur retour victorieux. Mais l'homme ne connait jamais son destin! Voici le texte du thème en luganda et en français:

\section{Abasiba embuzi \\ Basibira bwerere \\ A! Ssematimba ne Kikwabanga}

Ceux qui préparaient des chèvres

L'ont fait en vain

Ah! Ssematimba et Kikwabanga

Les harpistes développent ce texte de base en ajoutant des éléments narratifs et des explications. Musicalement, il est important que le texte de base et les variations suivent partiellement les inherent patterns qui surgissent du jeu instrumental - mais ce n'est pas systématique car l'interprète est libre de créer un grand nombre de variations. Il doit cependant observer un principe fondamental: les sons chantés doivent être à l'unisson ou à l'octave des notes instrumentales. En pratique, il y a certainement quelques anticipations et prolongations de la part du chanteur et ponctuellement de l'hétérophonie, mais cela ne viole pas le principe d'une musique essentiellement polyphonique où, a priori, toutes les combinaisons sonores se trouvent être des octaves ou des unissons.

La transcription de la figure 3 (p. 265) montre les deux principaux inherent patterns qui se dégagent de cette structure. Dans l'enregistrement (CD \# 3I) on peut entendre de quelle façon l'image acoustique se segmente pour recomposer des mélodies distinctes. Dans cette transcription, l'image acoustique totale consiste en la combinaison de tous les chiffres figurant sur les trois lignes. Dans la perception auditive, les sons sont réorganisés en deux couches selon un principe de proximité, 1 et 2 formant un premier groupe que l'attention perçoit, 5, 4 et 3 en formant un deuxième. À l'audition, l'attention peut se porter tantôt sur l'un tantôt sur l'autre.

L'inherent pattern composé des 1 et des 2 est l'image acoustique correspondant aux frappes des deux lames les plus graves (dites amatengezzi $)^{4}$. Le lecteur peut observer d'un coup d'œil comment le musicien 3, qui joue l'okukoonera obtient sa phrase complexe. Elle n'est rien d'autre que l'inherent pattern observé sur les amatengezzi, et ensuite joué par lui deux octaves plus haut sur les lames

[suite de la note 3] anachronisme sans conséquence parce qu'en vérité musicalement rien n’a changé en 33 ans, ainsi que l'atteste une vidéo récente.

4. Rappelons que chaque degré mélodique joué par les musiciens 1 et 2 est doublé à l'octave. L'I. P. 1 se dégage de la partie inférieure de l'octave. L'I. P. 2 se dégage, quant à lui, de la partie supérieure. 


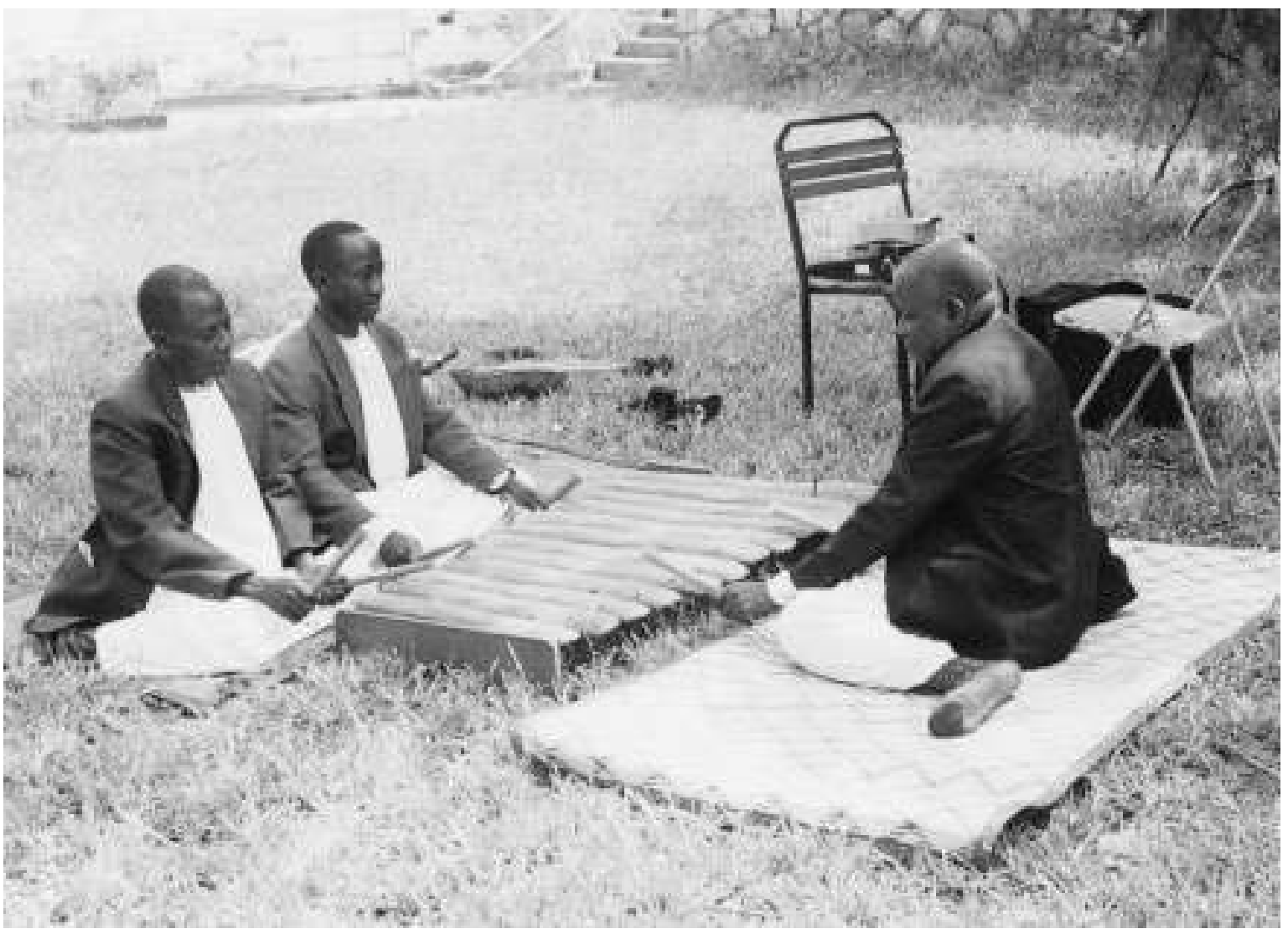

Jeu du xylophone amadinda à douze lames par le groupe d'Albert Ssempeke, 70 ans, musicien de cour en Buganda. Son fils Albert Bisaso frappe l'okunaga ; Albert Sempeke (à droite) prend la série okwawula, pendant que son frère, Ludowico Serwanga (à gauche) entonne l'okukoonera sur les lames les plus aiguës, Kampala, 6 février 2000 (Cl. Moya A. Malamusi). 


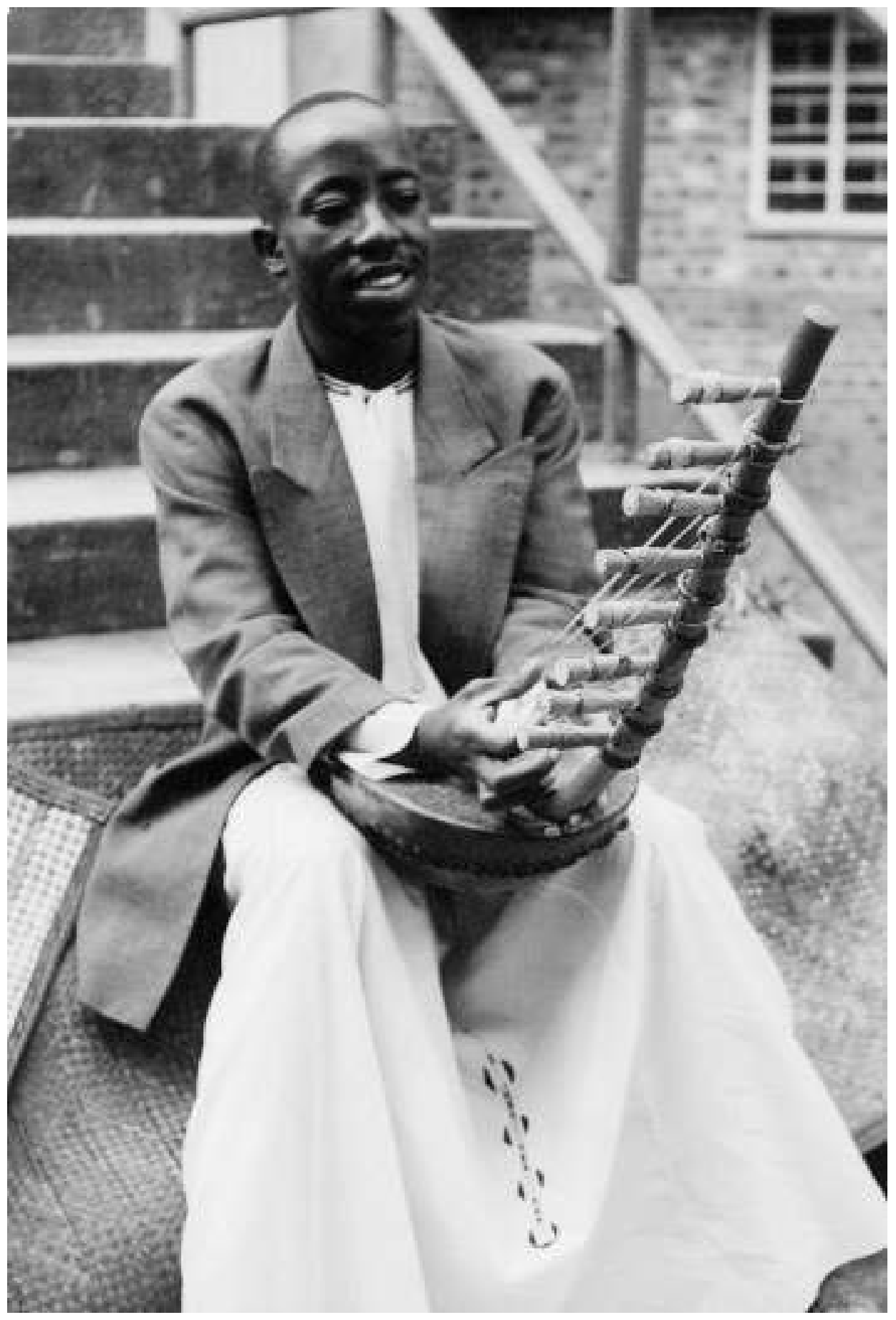

Le jeune Albert Bisaso, fils du musicien de cour Albert Ssempeke, jouant la composition Ganga alula sur une harpe ancienne ennanga à Kampala, 6 février 2000 (Cl. Moya A. Malamusi). 
les plus aiguës, dites amakoonezi (cf. CD \# 29). Il s'agit là d'un petit secret que mon professeur ne m’avait pas révélé au début de mon apprentissage.

Indirectement, la réalisation de l'okukoonera est la preuve que les inherent patterns sont perçus et conceptualisés par le musicien lui-même. C'est lui qui doit imiter l'inherent pattern le plus plus grave produit par les amatengezzi. Il $\mathrm{y}$ a aussi un aspect technique dans cette tâche car le troisième musicien doit apprendre à manœuvrer de façon légère et économique ses baguettes droite et gauche.

Ainsi pour chaque composition d'amadinda, le troisième musicien apprend d'abord comment alterner les frappes droites et gauches comme il faut. Alors que chacun des deux autres musiciens joue sa partie à deux mains simultanément en octaves parallèles, le troisième fait alterner ses deux mains. Il doit mémoriser la structure de la phrase et veiller à ce que toutes ses frappes soient synchronisées avec l'inherent pattern des amatengezzi, lequel est très audible et correspond aux notes les plus graves de l'instrument. Evaristo Muyinda attira souvent mon attention sur le fait que le troisième musicien doit bien écouter la suite de sons qui proviennent de ces deux lames en les imitant deux octaves plus haut sur les amakoonezi. Sa fonction est considérée comme la plus difficile. Déjà, du temps de mon apprentissage, les experts étaient rares. On comprend pourquoi. Comme il s'agit de dupliquer un inherent pattern deux octaves plus haut, le musicien doit avoir une bonne oreille et le reconnaitre pour l'extraire. Pour quelques compositions c'est possible sans trop d'effort, mais certaines sont très longues. La plus longue comprend un cycle de 70 unités de pulsation et s'appelle Agenda n’omulungi azaawa ("Qui se promène avec les beautés se perd»). Faire la partie okukoonera, c'est "frapper en relation avec quelque chose": a) en relation avec la phrase (l'inherent pattern) qui se perçoit à partir des deux lames de l'amatengezzi; b) en relation avec le schéma interne de la pulsation élémentaire. Si ce schéma est insuffisamment prégnant chez un musicien, il y aura un problème de coordination.

L'okukoonera comprend une séquence de frappes très irrégulières (CD \# 30 et Fig. 3 p. 265). La distribution des points d'impact de cette phrase semble sans structure. Elle semble ne pas être décomposable en segments répétés. Il restera toujours quelques moments syncopés. Pourtant la séquence n'est pas chaotique. En effet, elle est créée par un ensemble plus grand d'événements sonores. Il s'agit de l'image totale, qui peut être jouée en cinq transpositions différentes, qu'on appelle en luganda les emiko (pluriel, emiko, singulier omuko). L'okukoonera que j'ai transcrit (Fig. 3 p. 265) est celui du premier omuko (la première transposition) de la composition Ssematimba..., mais il y en a d'autres. Pour le moment il nous suffit de retenir trois principes qui le définissent : 1) il couvre 36 unités de pulsation ; 2) il est rigoureusement répété ; 3) chaque point d'impact coïncide avec une unité de pulsation.

Quand la phrase est jouée seule, pour la démonstration ou l'apprentissage, cette coïncidence n'est pas toujours nette, parce que l'exécutant se souvient de l'okukoonera plutôt comme une phrase parlée. Mais dès lors qu'il joue en 
ensemble avec ses partenaires, le réseau de références est psychiquement activé. La coordination avec la pulsation élémentaire devient plus rigoureuse, cadrée par l'attention portée aux amatengezzi.

Pourtant il peut y avoir des structures cachées dans un okukoonera. Par exemple dans l'omuko présent de la composition Ssematimba..., on peut découvrir des permutations d'ensembles de 2 et 3 pulsations, auxquelles s'ajoutent par quatre les unités additionnelles remplissant le cycle. La séquence est la suivante : $4+\mathrm{P}(332)+\mathrm{P}(332)+\mathrm{P}(323)+\mathrm{P}(323)=36$.

Reste à expliquer la théorie des emiko, qui est assez complexe. En bref, l'image de Ssematimba ne Kikwabanga que nous avons transcrite n'est pas la seule qui existe; il y a cinq images ou apparences de la même composition (au moins en théorie, en pratique on constate que les musiciens n'en jouent que deux). Chaque morceau d'amadinda peut donner lieu à cinq transpositions différentes. Et chacune donne des inherent patterns assez jolis, comme ceux que nous avons transcrits. Ce serait déborder de notre sujet que d'expliquer ici le formidable système de dérivées qu'utilise cette musique. Il suffit de mentionner qu'en principe chaque composition, y compris Ssematimba ne Kikwabanga, peut être jouée sous cinq "apparences» différentes. Littérairement emiko signifie «les plis» (par exemple d'un rideau) ou "les feuilles » pliées pour former un livre ou un journal. La composition est donc "pliée" cinq fois, mais les cinq plis sont structurellement identiques. La gestalt mélodique change, alors que la structure des mélodies est conservée. Ainsi la série 4.5.2.3.3.5.2.1. que l'on trouve dans la partie okunaga se transforme en 5.1.3.4.4.1.3.2 etc. dans la première transposition qui semble être une mélodie différente à cause du déplacement d'octaves. La deuxième transposition sera 1.2.4.5.5.2.4.3 etc., ici encore mélodiquement différente mais structurellement identique. C'est pourquoi Joseph Kyagambiddwa (1955) disait que chaque omuko a un caractère spécial. Chacun a une structure très organisée. Dans chaque cas, il y a un inherent pattern qui surgit des amatengezzi, I. P. que doit doubler le troisième musicien, alors qu'on peut aussi en discerner un deuxième dans l'arrière-plan de l'image totale.

En principe donc, chaque composition d'amadinda peut prendre cinq apparences différentes, sans que sa structure soit changée. Le plus étonnant c'est que dans les cinq cas, quelque chose de spectaculaire surgira des amatengezzi. La mathématique qui est derrière ces compositions, structurées comme des petits univers autonomes, reste encore à déchiffrer.

\section{Le domaine expérimental}

À l'époque de mes premières recherches au Buganda (1959-1960), je ne connaissais pas les études audio-psychologiques antérieures concernant la « fission » des mélodies; je n'avais aucune notion de la façon dont notre appareil perceptif réorganise un ensemble mélodique en lignes séparées. Dans ce domaine expérimental, le travail de George Heise et George Miller a précédé le mien. En 1947, George Miller découvrit par une expérience menée en laboratoire que des sons 
graves et aigus présentés en alternance rapide aux auditeurs créaient un effet de ségrégation en deux lignes séparées. Plus tard, George Miller et George Heise (Heise \& Miller 1951) cherchèrent à connaitre les conditions de cette ségrégation et à déterminer la largeur de l'intervalle requis pour qu'elle se produise (notion de trill threshold). À partir des années 1970, plusieurs collègues en psychologie de l'audition et en psychologie cognitive se sont occupés de ce phénomène (sans avoir cependant connaissance de mes études au Buganda), notamment Albert S. Bregman (1978, 1990), Bregman \& Jeffrey Campbell (1971), W. Jay Dowling (1973), Leo Paulu A. S. Van Noorden (1975). Parmi les travaux les plus récents, citons l'article de Carlton (Carlton et al. 2001). Tous se sont appuyés sur des méthodes classiques de laboratoire, offrant à leurs sujets (surtout occidentaux) des stimuli spécialement créés pour l'expérience.

En psychologie de l'audition, la formation des inherent patterns s'analyse en terme d'auditory stream segregation (ségrégation des courants auditifs) (Bregman \& Campbell 1971). Il s'agit de concevoir et de décrire comment notre appareil perceptif réagit à des bombardements de stimuli continuels. Dans ma propre terminologie, en 1960, je faisais état de couches superposées consistant en sons associés (en anglais pitch layers, en allemand Tonhöhenschichten). Dans le système tonal équipentatonique de la musique d'amadinda, la couche la plus basse inclut les sons des lames 1 et 2 et recouvre donc une bande d'environ 240 cents. Audessus se trouve la couche qui recouvre trois «hauteurs» $(3,4,5)$, constituant une bande perceptive d'environ 480 cents. Les deux couches sont rigoureusement séparées; il y a une frontière entre les deux qui est immuable. Les octaves parallèles renforcent ce dispositif.

Que l'on parle de "couches" (layers) ou de "courants» (streams) il est évident qu'il s'agit d'un même phénomène. Pourtant, il y a des différences d'approches entre des recherches sur le terrain en Afrique et les recherches purement expérimentales réalisées en laboratoire: Miller, Heise, Bregman, Dowling et d'autres ont toujours créé des conditions d'observation caractéristiques de la méthode expérimentale. Dans cette tradition scientifique, on opte souvent pour un système à deux variables, que l'expérimentateur peut manipuler (par exemple, en changeant les fréquences d'un son) en restant attentif aux réactions des sujets; on s'efforce d'éliminer le plus grand nombre possible de facteurs dérangeants afin d'arriver à des relations logiques de cause à effet.

Il est évident que, sur le terrain, la situation est différente. D'abord je découvris au Buganda, plus ou moins par hasard, une culture musicale qui avait existé durant une longue période historique et dans laquelle les anciens compositeurs avaient créé des structures sonores que la perception auditive humaine sépare de la même façon que les patterns créés expérimentalement dans les laboratoires d'Europe et d'Amérique; avec une différence notable cependant : ces structures dans la musique de Buganda étaient beaucoup plus complexes que celles que l'on concevait en laboratoire. Sur le terrain, j'adoptai donc une méthode interactive incluant l'observation de mes partenaires et l'introspection, sans exclure de recourir plus tard à des expériences plus synthétiques. 
Cette phase s'enclencha grâce aux travaux de mon collègue de Berlin, Ulrich Wegner, qui créa en 1987 des versions synthétiques (modélisées sur ordinateur) de quelques compositions réalisées à partir de mes transcriptions (Wegner $1990,1993)$. Les résultats furent très convaincants. Wegner a pu montrer entre autre que :

a) sur la base de reconstructions synthétiques et d'enregistrements sur le terrain du jeu original, les réactions de l'appareil perceptif humain sont les mêmes, on entend toujours les mêmes inherent patterns;

b) si dans un enregistrement stéréo, l'okunaga est retransmis dans une oreille et l'okwawula dans l'autre, le sujet est incapable d'intégrer les deux ;

c) il n'y a pas d'intégration non plus lorsque les deux parties sont distantes d'une octave.

En 1959-1960, je réalisai que les ancêtres compositeurs de cette musique devaient avoir des connaissances intimes, totalement empiriques, sur le fonctionnement de notre appareil perceptif, pour avoir créé de telles compositions. La jeune génération ne les avait plus, et peu de compositions récentes étaient comparables en complexité avec le répertoire "classique " remontant au moins à la fin du XVIII siècle. C'était vrai aussi pour les pièces de xylophone jouées au Busoga, État voisin de Buganda (Kubik 1992). Ma recherche sur le terrain se développa alors dans des conditions où il était possible d'étudier les concepts et le comportement actuels des exécutants de cette musique, en écoutant leur musique; toute conclusion sur le processus de composition d'autrefois restait hypothétique. J'ai tout de même pu, à partir de la terminologie contemporaine des musiciens en langue luganda, arriver à des conclusions concernant le savoirfaire des anciens compositeurs et comprendre, sur la base des sons produits, comment ils savaient utiliser les ressources de l'appareil perceptif humain.

Dès le début de mes recherches, $j$ 'avais observé que deux principes fondamentaux étaient nécessaires pour la création d'inherent patterns et d'une gestalt auditive : celui de la proximité (selon le système tonal de la région, notes distantes au minimum de 240 cents et au maximum de 480 cents); et celui de la similarité de timbre (Kubik 1960 : 13). Bien sûr, il s'agit là d'un principe de similarité et non d'une identité totale! Au Buganda, on ne recherche pas un timbre uniforme pour toutes les lames d'un xylophone, mais on souligne l'individualité de chaque note. Comme on l'a déjà dit, l'effet de fusion acoustique est limité par un accordage altérant la relation d'octaves d'environ 10 à 15 cents.

Ces deux principes étaient en accord avec la théorie d'association telle qu'elle était formulée par les psychologues de la Gestalt. Il y avait cependant ici un petit élément nouveau, qui concernait le principe de proximité, cette notion devant toujours être comprise dans son sens relatif, en fonction du système tonal de la culture musicale étudiée. Sur un piano, la proximité maximale de deux sons est de 100 cents, mais sur l'amadinda elle est d'environ 240 cents. Ce système tonal constitue un cadre culturel invariable pour 
les stimuli auxquels l'individu est exposé. En accord avec d'autres principes d'association formulés par ces mêmes psychologues, j'ai aussi noté l'existence d'autres conditions pour que l'effet I. P. se produise:

1) les intervalles (pentatoniques dans ce cas) doivent être assez larges, pour que l'image totale se sépare en deux;

2) la pièce jouée doit l'être rapidement pour créer un tel effet;

3) sa structure ne doit pas avoir de forts accents métriques (sans quoi elle formerait une autre ligne de référence);

4) la pièce doit être organisée sous une forme rythmiquement complexe; l'emploi des schémas métriques simples semble diminuer l'effet I. P. sans pourtant l'éliminer complètement (Kubik 1962:36).

Finalement, je mentionnai que l'effet I. P. se trouve occasionnellement dans la musique baroque, notamment chez Jean-Sébastien Bach, malgré une certaine régularité métrique qui n'existe pas dans mes exemples africains.

Dans un article plus récent (Kubik 1989 : 133), j’ai repris la question des conditions nécessaires pour qu'une mélodie se sépare en deux en mettant l'accent sur le fait que la manifestation de l'effet I. P. doit être mise en relation avec la nature du système tonal utilisé. Par exemple, la bande la plus large dans la musique de Buganda ne dépasse pas 480 cents (soit environ, une quarte) et la limite minimum 240 cents (une seconde un peu large). Dans la musique du lamellophone likembe, dans l'Est de l'Angola, on est dans un système hexa/heptatonique comprenant des intervalles plus serrés. À l'évidence, la largeur des intervalles d'un système joue un rôle premier pour déterminer les frontières entre les couches perceptibles possibles. Je mentionnai aussi dans cet article que des facteurs auxiliaires favorisaient ce phénomène de fission, et que les compositeurs anciens au Buganda et ailleurs devaient les connaître : en donnant, par exemple, la préférence à certains ordres numériques dans l'enchaînement des notes, en utilisant la pulsation élémentaire pour donner une certaine densité à l'image auditive, en favorisant une irrégularité des séquences mélodiques et en pratiquant la répétition cyclique.

En ce qui concerne la vitesse d'exécution, le lecteur peut faire ses propres expériences à partir des enregistrements qui accompagnent le présent travail. Au Buganda, en notant ce qui se passait quand je reproduisis mes enregistrements à mi-vitesse sur mon magnétophone (une octave plus basse), j'avais observé que le tempo était un facteur important. À mi-vitesse, les inherent patterns étaient encore discernables, la pulsation élémentaire se déroulait à 300 pulsations à la minute, mais si on abaissait encore la hauteur d'une octave les patterns disparaissaient. Malheureusement je n'avais pas alors la possibilité de changer le tempo sans changer les fréquences et il n'était donc pas possible d'éliminer la variable de registre. Mais Leo Van Noorden publia en 1975 les résultats de ses expériences par lesquelles il put séparer les deux facteurs concomitants que je n’avais pu séparer: le registre et le tempo.

Le phénomène de formation d'inherent patterns (alias auditory streaming), sujet d'une de mes premières recherches en Afrique, a suscité un intérêt considérable dans le domaine de la psychologie de l'audition et de l'ethnomusicologie 
cognitive. Cet intérêt ne se limite d'ailleurs pas à la recherche scientifique. Des compositeurs non africains ont expérimenté ces techniques et ont pu construire des effets d'illusions auditives ${ }^{5}$.

Sur le plan scientifique, mes expériences africaines et interculturelles suggèrent qu'il s'agit d'une aptitude auditive universelle qui peut être utilisée et développée chez l'individu à l'intérieur d'un environnement culturel favorable. J'ai ainsi pu constater que, historiquement, l'utilisation de l'effet I. P. dans la composition musicale se concentre surtout dans quelques régions de l'Afrique orientale et centrale, notamment au Buganda, dans l'ancienne musique de cour du royaume. Je pense même que l'apogée de ces techniques et connaissances au Buganda fut située à la fin du XVIII et au XIX ${ }^{\mathrm{e}}$ siècle, sous les règnes de Ssekabaka Kamaanya, puis de Suuna II (1826-1854) et de Mutesa I (18541885), lequel jouait lui-même de l'amadinda, ainsi que d'autres instruments ${ }^{6}$. Je pense aussi que la fission et la reconstitution d'une image auditive relèvent d'une réaction compensatrice de notre appareil perceptif (Kubik 1989 : 37). Il est évident que les images complexes de cette musique de cour étaient intentionnellement créées, peut-être pour des raisons touchant au religieux, car le caractère méditatif du jeu de la harpe ennanga est indéniable et que les inherent patterns semblent "parler" au musicien lorsqu'il est seul, comme la voix d'un esprit.

Les anciens compositeurs royaux de Buganda avaient probablement plusieurs raisons de créer des images acoustiques qui se séparent en deux lignes, résultats d'une saturation perceptive créant une désorientation qui est ensuite compensée en une fraction de seconde: notre appareil auditif réorganise l'ensemble de la musique, reconstituant ainsi des ensembles complexes perceptibles. Le fait que la musique soit structurée d'une certaine façon facilite le processus. C'est à ce résultat que les anciens compositeurs de Buganda sont parvenus. Et tandis que la réorganisation perceptive se fait selon les principes de proximité, de similarité, etc., les inherent patterns eux-mêmes ne sont en rien une chimère. Ils sont inhérents à la composition et sont présents dans la création des compositeurs. Ces derniers ont appris empiriquement comment manipuler l'appareil perceptif pour créer de tels effets qu'un harpiste d'ennanga en méditation, pour sa part, perçoit comme si c'était les voix d'esprits parlant de l'intérieur de son instrument.

Je ne crois pas que l'explication de l'effet I. P. soit donnée dans une théorie de la psychologie de l'audition, fréquemment citée, selon laquelle la cause de ce phénomène serait à chercher dans les différents groupements de fréquences qui caractérisent l'organisation de notre système auditif. Ma propre explication est

5. Notamment Györgi Ligeti : cf L'Escalier du diable Édition SK 62308, exécuté au piano par Pierre-Laurent Aimard, CD, Schott Musik International, Mainz, 1996. Ligeti n'a pas emprunté directement à la musique africaine, mais explore plutôt des chemins analogues créant des images acoustiques paradoxales.

6. L'enregistrement le plus ancien d'amadinda a été réalisé sur cylindre par Sir Harry Johnston vers 1900. J'ai pu identifier la composition et constater qu'elle est identique à celle que j'ai notée en 1959-1960 en travaillant avec Evaristo Muyinda. 
voisine de celle de Miller (1956), qui avait parlé d'une channel capacity de l'appareil perceptif humain. En plus, selon moi, si l'effet I. P. est comparable à des effets similaires dans le domaine de la perception visuelle, il est probable que l'organisation de la perception auditive et celle de la perception visuelle sont structurellement analogues (voir quelques illusions d'optique créées par Gaetano Kanisza). Il s'agit là d'un champ d'investigation auquel je souhaite qu'on prête plus d'attention à l'avenir.

MOTS CLÉS/KEYWORDS: royaume de Buganda/Kingdom of Buganda - psychologie cognitive/cognitive psychology - inherent patterns - xylophone amadindalamadinda xylophoneségrégation des courants auditifs/auditory stream segregation - notation chiffrée/ figured notation.

\section{BIBLIOGRAPHIE}

Bregman, Albert S.

1978 « Auditory Streaming: Competition among Alternative Organizations ", Perception and Psychophysics 23 (5): 391398.

1990 Auditory Scene Analysis: The Perceptual Organization of Sound. Cambridge, MIT Press.

\section{Bregman, Albert S. \& Jeffrey Campbell}

1971 «Primary Auditory Stream

Segregation and Perception of Order in Rapid Sequences of Tones ", Journal of Experimental Psychology 89 (2) : 244-249.

Carlton, R. P. et al.

2001 "Effects of Attention and Unilateral Neglect on Auditory Stream Segregation ", Journal of Experimental Psychology 27 (1) : 115-127.

Dowling, W. Jay

1973 «The Perception of Interleaved Melodies ", Cognitive Psychology 5 : 322-337.

\section{Ehrenfels, Christian Freiherr von}

1890 "Über Gestaltqualitäten ", Vierteljahresschrift für Wissenschaftliche Philosophie 14 : 242-292.

\section{Gazzaniga, Michael S.}

1998 The Mind's Past. Berkeley, University of California Press.

Heise, George A. \& George A. Miller

1951 «An Experimental Study of Auditory Patterns ", American Journal of Psychology 64 (1) : 68-77.

Jones, Arthur M.

1934 «African Drumming: A Study in the Conbination of Rhythms in African Music ", Bantu Studies 8 : 1-16.

Koffka, Kurt

1935 Principles of Gestalt Psychology.

New York, Harcourt, Brace \& Company.

Köhler,Wolfgang

1929 Gestalt Psychology. New York, Liveright.

Kubik, Gerhard

1960 "The Structure of Kiganda

Xylophone Music ", African Music 2 (3):

6-30 [Corrigenda, 1970, African Music

4 (4) : 136-137].

1962 «The Phenomenon of Inherent Rhythms in East and Central African 
Instrumental Music ", African Music 3 (1) : 33-42.
Music ", Cross rhythms 3, Bloomington, Indiana University: 129-154.

1992 «Embaire Xylophone Music of

Samusiri Babalanda (Uganda 1968), The

World of Music 34 (1) : 57-84.

1994 [1969] Theory of African Music .

Vol. 1,Wilhelmshaven, Florian Noetzel.

Kyagambiddwa, Joseph

1955 African Music from the Source of the

Nile. New York, Pareger.

\section{Miller, George A.}

1956 «The Magical Number Seven, Plus or Minus Two : Some Limits on our Capacity of
Processing Information ", The Psychological Review 63 : 81-97.

Van Noorden, Leo Paulus Antonio Servatius

1975 Temporal Coherence in the Perception of Time Sequences.Eindhoven, Institute for Perception Research.

Wegner, Ulrich

1990 Xylophonmusik aus Buganda (Ostafrika), Musikbogen-Wege zum Verständnis fremder Musikkulturen. Wilhemshaven, Florian Noetzel (Avec cassette).

1993 " Cognitive Aspects of Amadinda Xylophone Music from Buganda : Inherent Patterns Reconsidered ", Ethnomusicology 37 (2) : 201-241.

RÉSUMÉ/ABSTRACT

Gerhard Kubik, Inherent patterns. - Une connaissance approfondie et une pratique de la musique de xylophone de l'ancien royaume de Buganda permettent à l'auteur de dégager certains principes de composition du "battement encrené», procédant par addition de pulsations rapides exécutées par (au moins) deux musiciens en une stricte alternance, chacun d'entre eux jouant, en décalage, sa propre série mélodico-rythmique. De cette technique découlent des «inherent patterns»: figures mélodiques entendues (en fait, re-composées mentalement) lors de l'audition et relevant d'une gestalt auditive. La thèse de l'auteur est que ces mélodies ont été intentionnellement créées par des compositeurs de la fin du XVIII e siècle disposant d'un savoir ésotérique supposant de grandes compétences sur les phénomènes de perception musicale.
Gerhard Kubik, Inherent Patterns. — Thanks to his in-depth knowledge and practice of the xylophone music of the former Kingdom of Buganda, the author has brought out certain principles of composition of « in-between " hammerings (an addition of fast pulsations, executed by at least two musicians in strict alternation, each of them playing, at different intervals, his own melodic, rhythmic series). "Inherent patterns » arise out of this technique: melodic figures heard (actually, recomposed mentally while listening) and referred to an auditory gestalt. The hypothesis is made that late 18th century composers, who had an esoteric knowhow and proficiency in the perception of music, intentionally created these melodies. 
MUSICIENS

\begin{tabular}{ll|l|l|l|l|l|l|l|l|l|l|l|l|l|l|ll} 
Musicien 3 & $\mathrm{~d}$ & $\mathrm{~d}$ & $\mathrm{~g}$ & $\mathrm{~d}$ & $\mathrm{~d}$ & $\mathrm{~g}$ & $\mathrm{~d}$ & $\mathrm{~g}$ & $\mathrm{~d}$ & $\mathrm{~g}$ & $\mathrm{~d}$ & $\mathrm{~g}$ & $\mathrm{~d}$ & $\mathrm{~d}$ & $\mathrm{~g}$ & $\mathrm{~d}$ & $\mathrm{~g}$ \\
okukoonera & $\overline{1}$ & $\overline{2}$ & $\overline{1}$ & $\overline{2}$ & $\overline{2}$ & $\overline{1}$ & $\overline{2}$ & $\overline{2}$ & $\overline{2}$ & $\overline{2}$ & $\overline{2}$ & $\overline{1}$ & $\overline{2}$ & $\overline{2}$ & $\overline{1}$ & $\overline{1}$ & $\overline{1}$
\end{tabular}

\begin{tabular}{|c|c|c|c|c|c|c|c|c|c|c|c|c|c|c|c|c|c|}
\hline Musicien 14 & (5) & 2 & (3) & (3) & (5) & 2 & 1 & 2 & (5) & 2 & 2 & 1 & (4) & (4) & 2 & 1 & 1 \\
\hline okunaga & $\underline{5}$ & $\underline{2}$ & $\underline{3}$ & $\underline{3}$ & $\underline{5}$ & $\underline{2}$ & 1 & $\underline{2}$ & $\underline{5}$ & \begin{tabular}{|l}
$\underline{2}$ \\
\end{tabular} & $\underline{2}$ & 11 & $\underline{4}$ & $\underline{4}$ & \begin{tabular}{|l}
2 \\
\end{tabular} & 1 & 1 \\
\hline
\end{tabular}

\begin{tabular}{ll|l|l|l|l|l|l|l|l|l|l|l|l|l|l|l|l|l} 
Musicien 2 & 1 & (4) & $(3)$ & 1 & 2 & (3) & $(4)$ & $(3)$ & 2 & 2 & $(5)$ & $(4)$ & $(3)$ & 2 & 4 & (4) & 4 & 1
\end{tabular} \begin{tabular}{l|l|l|l|l|l|l|l|l|l|l|l|l|l|l|l|l|l|l|} 
okwawula & $\underline{1}$ & $\underline{4}$ & $\underline{3}$ & $\underline{1}$ & $\underline{2}$ & $\underline{3}$ & $\underline{4}$ & $\underline{3}$ & $\underline{2}$ & $\underline{2}$ & $\underline{5}$ & $\underline{4}$ & $\underline{3}$ & $\underline{2}$ & $\underline{4}$ & $\underline{4}$ & $\underline{4}$ & $\underline{1}$ \\
\hline
\end{tabular}

\section{INHERENT PATTERNS}

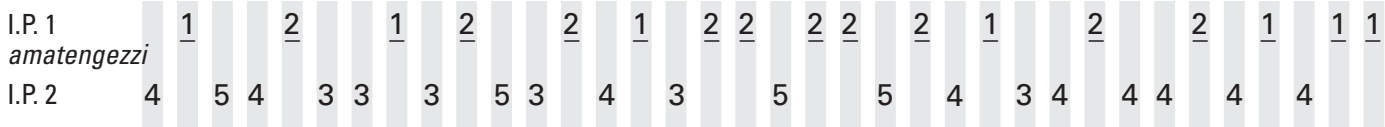

\section{THÈME CHANTÉ}

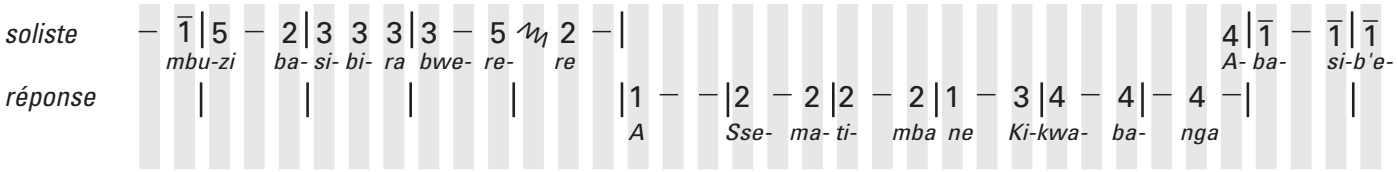

Figure 3

Transcription de la composition Ssematimba ne Kikwabanga - CD \# 31 et \# 32 - comprenant deux inherent patterns, plus ou moins bien perçus selon le timbre des instruments. Le tempo, très rapide, est de 600 pulsations à la minute, chaque case grise correspondant à une pulsation.

L'okunaga et l'okwawula sont joués en octaves parallèles.

Les I. P. 1 et 2 se dégagent de la combinaison de ces deux parties. L'I. P. 1. [degrés encadrés dans la figure] n'utilise que les degrés inférieurs de ces octaves parallèles ; l’I. P. 2 [degrés encerclés] n’utilise que les degrés supérieurs.

Les chiffres 1, 2, 3, 4, 5 renvoient aux degrés de l'échelle équipentatonique.

Chiffre souligné $=$ octave inférieure

Chiffre surligné = octave supérieure

Non souligné $=$ registre médian

$\mathrm{d}=$ baguette droite $\mathrm{du}$ troisième musicien

$\mathrm{g}=$ baguette gauche du troisième musicien

Les flèches signalent les points d'entrée des différents musiciens

Amatengezzi $=$ les deux lames les plus graves de l'instrument Amakoonezi = les deux lames les plus aiguës de l'instrument 